\title{
Anomalies de TrkA, le récepteur du NGF, chez les malades atteints d'insensibilité congénitale à la douleur
}

Les patients atteints d'insensibilité congénitale à la douleur (ICD) présentent un tableau clinique très particulier: la sensation douloureuse et, dans une moindre mesure, la sensibilité thermique sont fortement altérées alors que les autres modalités sensorielles sont intactes; ils ont une espérance de vie plus courte car ils sont sujets à des traumatismes dont ils n'ont pas conscience et qui entraînent souvent, de ce fait, de graves complications. Cette impossibilité de ressentir la douleur conduit à de fréquentes blessures graves, pouvant aller jusqu'à la perte de doigts ou à des mutilations sévères de la langue [1]. La première autopsie réalisée d'une ICD a permis de mettre en évidence une neuropathie sensorielle associée à l'absence totale, d'une part des neurones parvocellulaires des ganglions des racines dorsales (GRD), d'autre part des petites fibres dans les racines dorsales et le tractus de Lissauer, ainsi qu'une réduction de la taille du tractus spinal du nerf trijumeau. Les ICD sont maintenant classées en cinq types de neuropathies sensorielles héréditaires et l'examen au microscope de biopsies des nerfs périphériques a révélé, pour chaque type, l'absence de petites fibres $\mathrm{A} \delta$ et/ou $\mathrm{C}$, accompagnée ou non d'une dégénérescence des neurones des GRD. L'une d'entre elles (ICD de type IV, maladie autosomique récessive) est caractérisée par l'absence de réaction aux stimulus nociceptifs, un comportement d'automutilation et un retard mental, ainsi que par une anhydrose (absence totale de sudation) liée à

thique des glandes exocrines sudoripares et associée à des épisodes récurrents inexpliqués de fièvre [2]. Le NGF (nerve growth factor) découvert il y a un demi-siècle est un facteur de survie sécrété par les tissus cibles du système nerveux périphérique (sensoriel nociceptif et sympathique), dont dépend l'intégrité des fibres de ces systèmes (fibres $\mathrm{A} \delta$ et $\mathrm{C}$ et fibres sympathiques) et des corps neuronaux dont elles proviennent, riches en récepteurs TrkA à forte affinité pour le NGF. En accord avec l'importance du rôle du NGF et de ses récepteurs dans la maturation et l'activité des neurones sensoriels nociceptifs, les animaux déficients en récepteurs à forte (TrkA) et à basse (LNGFR) affinité présentent une absence de réponse comportementale aux stimulus nociceptifs associée à une perte très importante des neurones sensoriels parvocellulaires du GRD [3, 4]. Ces résultats obtenus par recombinaison homologue confirmaient ceux plus anciens utilisant des anticorps anti-NGF, injectés précocement au cours du développement pour neutraliser l'action du NGF, qui conduisaient à une perte substantielle du nombre de neurones des GRD.

Forte de ces données, une équipe japonaise a recherché une atteinte du signal NGF ou de sa réception chez trois patients non apparentés, issus de parents consanguins, atteints d'ICD de type IV [5]. Utilisant la propriété des lymphocytes d'exprimer les gènes $N G F$ et TRKA, cette équipe a utilisé des cellules lymphoblastoïdes transformées par le virus d'Epstein-Barr, issues de ces trois patients et de membres de leurs familles, pour extraire de l'ARNm amplifié ensuite par PCR. Ils ont ainsi caractérisé chez ces trois patients trois mutations différentes, détectées sur l'ARNm et confirmées sur l'ADN génomique, dans la séquence codant pour le domaine tyrosine kinase de TrkA: une délétion, une mutation non-sens et une mutation d'épissage. Ces résultats suggèrent fortement que l'ICD de type IV trouve son origine dans ces mutations de TrkA, avec perte de sa fonction. Cela confirme que chez l'homme le système TrkA-NGF joue le même rôle déterminant dans le développement et le fonctionnement des systèmes nociceptifs que chez la souris. De plus, ce système NGF/TrkA semble impliqué dans le développement ou la fonction des systèmes de thermorégulation et de contrôle de la sudation.

B.C.

1. Sweet WH. Animal models of chronic pain: their possible validation from human experience with posterior rhizotomy and congenital analgesia. Pain 1981; 10 : 275-95.

2. Swanson AG. Congenital insensitivity to pain with anhidrosis. Arch Neurol 1963; 12: 12-8.

3. Smeyne RJ, Klein R, Schnapp A, Long LK, Bryant S, Lewin A, Lira SA, Barbacid M. Severe sensory and sympathetic neuropathies in mice carrying a disrupted Trk/NGF receptor gene. Nature 1994; 368: 246-9.

4. Onteniente B. Mieux comprendre le rôle des neurotrophines dans le système nerveux grâce à l'invalidation génique. Med Sci 1995; 11: 1141-3. 5. Indo Y, Tsuruta M, Hayashida Y, Karim MA, Ohta K, Kawano T, Mitsubushi H, Tonoki H, Awaya Y, Matsura I. Mutations in the TrkA/NGF receptor gene in patients with congenital insensitivity to pain with anhidrosis. Nature Genet 1996 ; 13: 485-8. 